PROCEEDINGS OF THE

AMERICAN MATHEMATICAL SOCIETY

Volume 126, Number 8, August 1998, Pages 2507-2508

S 0002-9939(98)04691-7

\title{
A SHORT PROOF OF A CHARACTERIZATION OF REFLEXIVITY OF JAMES
}

EVE OJA

(Communicated by Dale Alspach)

\begin{abstract}
A short direct proof is given to a well-known intrinsic characterization of reflexivity due to $\mathrm{R}$. C. James.
\end{abstract}

The following famous intrinsic geometric characterization of reflexivity is due to R. C. James [4] (cf. also e.g. [1, p. 51], [2, p. 58] or [3]).

Theorem. A Banach space $X$ is reflexive if and only if there is a $\theta \in(0,1)$ such that if $\left(x_{n}\right)_{n=1}^{\infty}$ is a sequence of elements of the unit sphere of $X, S_{X}$, with $\|u\|>\theta$ for all $u \in \operatorname{conv}\left\{x_{1}, x_{2}, \ldots\right\}$, then there are $n_{0} \in \mathbb{N}, u \in \operatorname{conv}\left\{x_{1}, x_{2}, \ldots, x_{n_{0}}\right\}$ and $v \in \operatorname{conv}\left\{x_{n_{0}+1}, x_{n_{0}+2}, \ldots\right\}$ such that $\|u-v\| \leq \theta$.

This note provides a short and easy direct proof of the James theorem. It does not rely on Helly's theorem (like the proof in [4]) nor the Šmulian-Eberlein theorem (like the proof in [5, pp. 95-99]).

Proof of the Theorem. Necessity. The following proof is traditional. We present it here for the sake of completeness. Let $X$ be reflexive. Fix any $\theta>0$, consider any $\left(x_{n}\right)_{n=1}^{\infty} \subset S_{X}$, and denote $K_{n}=\overline{\operatorname{conv}}\left\{x_{n+1}, x_{n+2}, \ldots\right\}$. Since $\left(K_{n}\right)_{n=0}^{\infty}$ is a nested sequence of weakly compact sets, there is $x \in \bigcap_{n=0}^{\infty} K_{n}$. Since $x \in K_{0}$, there are $n_{0} \in \mathbb{N}$ and $u \in \operatorname{conv}\left\{x_{1}, \ldots, x_{n_{0}}\right\}$ such that $\|x-u\|<\theta / 2$. Since $x \in K_{n_{0}}$, there is $v \in \operatorname{conv}\left\{x_{n_{0}+1}, x_{n_{0}+2}, \ldots\right\}$ such that $\|x-v\|<\theta / 2$. Hence $\|v-u\|<\theta$.

Sufficiency. Denote $B_{\theta}=\left\{F \in X^{* *}:\|F\| \leq \theta\right\}$. This is a weak ${ }^{*}$ closed set. If $X \neq X^{* *}$, then by Riesz's lemma there is $F_{\theta} \in S_{X^{* *}} \backslash\left(B_{\theta}+\{x\}\right)$ for all $x \in X$. Note that $F_{\theta}$ is in the weak* closure of $S_{X}$ (by Goldstine's theorem and weak* lower-semicontinuity of the norm). Pick any $x_{0} \in S_{X}$. Since the weak* open set $X^{* *} \backslash\left(B_{\theta}+\left\{x_{0}\right\}\right)$ contains $F_{\theta}$, it also contains a convex weak ${ }^{*}$ neighbourhood $V_{1}$ of $F_{\theta}$, which means

$$
\left\|v-x_{0}\right\|>\theta \quad \forall v \in V_{1} .
$$

Since $F_{\theta} \in X^{* *} \backslash B_{\theta}$, we can assume that $V_{1} \subset X^{* *} \backslash B_{\theta}$. Pick any $x_{1} \in V_{1} \cap S_{X}$. Since $F_{\theta} \in X^{* *} \backslash\left(B_{\theta}+\operatorname{conv}\left\{x_{0}, x_{1}\right\}\right)$, there is a convex weak* neighbourhood $V_{2} \subset$ $V_{1}$ of $F_{\theta}$ such that

$$
\|v-u\|>\theta \quad \forall u \in \operatorname{conv}\left\{x_{0}, x_{1}\right\}, \quad \forall v \in V_{2} .
$$

Received by the editors June 20, 1997 and, in revised form, August 27, 1997.

1991 Mathematics Subject Classification. Primary 46B20.

(C) 1998 American Mathematical Society 
Pick any $x_{2} \in V_{2} \cap S_{X}$ and continue as above. The sequences of convex sets $V_{1} \supset V_{2} \supset \cdots$ and elements $\left(x_{n}\right)_{n=1}^{\infty} \subset S_{X}$ satisfy $x_{n} \in V_{n}$ and

$$
\|v-u\|>\theta \quad \forall u \in \operatorname{conv}\left\{x_{1}, \ldots, x_{n}\right\}, \quad \forall v \in V_{n+1} .
$$

Since $\operatorname{conv}\left\{x_{n}, x_{n+1}, \ldots\right\} \subset V_{n} \subset X^{* *} \backslash B_{\theta}$, this contradicts the assumption.

\section{REFERENCES}

1. B. Beauzamy, Introduction to Banach spaces and their geometry, North-Holland Math. Stud., vol. 68, North-Holland, Amsterdam, 1982. MR 84g:46017

2. S. Guerre-Delabrière, Classical sequences in Banach spaces, Marcel Dekker, New York, 1992. MR 94d:46013

3. R. Haller and E. Oja, Geometric characterizations of positions of Banach spaces in their biduals, Arch. Math. 69 (1997), 227-233. CMP 97:16

4. R. C. James, Weak compactness and reflexivity, Israel J. Math. 2 (1964), 101-119. MR 31:585

5. V. D. Milman, Geometric theory of Banach spaces II, Uspekhi Mat. Nauk 26 (6) (1971), 73-149. (Russian) MR 54:8240

Faculty of Mathematics, Tartu University, Vanemuise 46, EE2400 Tartu, Estonia

E-mail address: eveoja@math.ut.ee 